\title{
Stent flexibility: an essential feature in the treatment of dynamic airway collapse
}

\author{
H. Hautmann, R.M. Huber
}

Stent flexibility: an essential feature in the treatment of dynamic airway collapse. $H$. Hautmann, R.M. Huber. CERS Journals Ltd 1996.

ABSTRACT: Implantation of endobronchial stents for treatment of dynamic airway collapse represents a suitable therapeutic option to alleviate distressing symptoms.

We report the case of a 43 year old patient suffering from progressive respiratory distress 2 weeks after insertion of a balloon-expandable radial noncompliant Palmaz stent in an unstable segment of the left main bronchus, with the aim of preventing symptomatic airway collapse. Bronchial instability had developed following sleeve resection of the right lung due to adenoid cystic carcinoma.

Explantation revealed compression and deformation of the stent. Peak expiratory flow (PEF) had declined to a low of $1.38 \mathrm{~L} \cdot \mathrm{s}^{-1}$ (forced expiratory volume in one second (FEV1) 1.02 L). With placement of a Strecker stent, having the ability to re-expand within certain limits, bronchial collapse could be avoided and marked clinical improvement as well as expiratory flow increase was noted $\left(P E F 7.10 \mathrm{~L} \cdot \mathrm{s}^{-1}\right.$; FEV1=2.03 L). At 13 months follow-up, clinical status was unchanged. A decline in forced expiratory flow (PEF 5.96 L·s $\mathrm{s}^{-1}$; FEV1 1.69 L), however, indicated a possible change in the structural integrity of the Strecker stent.

We conclude that physical properties of endobronchial stents may be crucial for good functional results in major airway collapse. Stiff prostheses, when compressed, can induce severe airway obstruction.

Eur Respir J., 1996, 9, 609-611.
Medizinische Klinik, Klinikum Innenstadt, Ludwig-Maximilians-University, Munich, Germany.

Correspondence: H. Hautmann

Klinikum Innenstadt

Medizinische Klinik

Ziemssenstr. 1

D - 80336 München

Germany

Keywords: Airway obstruction therapy anastomosis

bronchial diseases therapy

elasticity

expandable metallic stents

prosthesis design

Received: December 271994

Accepted after revision October 141995
The implantation of prosthetic tracheobronchial metal stents has been successfully carried out in benign tracheobronchial disease [1-4]. This report focuses on a case of dynamic airway collapse secondary to bronchial instability treated with two different types of balloonexpandable endobronchial prostheses, the Palmaz-Stent [5] and the Strecker-Stent [6]. In order to achieve acceptable functional results, design and properties of the prostheses had to meet the demands of the affected airway, particularly with regard to flexibility and stability. Although physiologic studies of tracheobronchial metal stents in airway obstruction have been carried out $[7,8]$, no data about strain and damage-tolerance within collapsing airways are yet available.

\section{Case history}

A 43 year old man has been treated with sleeve pneumonectomy due to adenoid cystic carcinoma. Three and a half years later, bronchial instability developed at the site of the tracheobronchial anastomosis, with total collapse of the airway during cough procedures. This led to marked reduction in expiratory flow rates. Vital capacity (VC), forced expiratory volume in one second (FEV1) and peak expiratory flow rates (PEF) were measured in a body plethysmograph (Bodyscreen, Jaeger, Würzburg, Germany) and are shown in table 1. The flow-volume loop illustrates the increased expiratory flow limitation at forced expiration (fig. 1a and b). Histopathology demonstrated inflammatory granulomatous reaction, without signs of recurrent tumour.

As a result of the inability to perform adequate mucus clearance, a Palmaz-Stent (Johnson \& Johnson Interven-

Table 1. - The course of pulmonary function

\begin{tabular}{lccc}
\hline & VC & FEV1 & PEF \\
& L & L & L·s ${ }^{-1}$ \\
\hline 9 months postoperative & 3.26 & 2.89 & 9.32 \\
45 months postoperative, before & 2.98 & 1.12 & 2.20 \\
Palmaz stent implantation when & & & \\
bronchial collapse was present & & & \\
14 days after Palmaz stent implantation & 2.77 & 1.02 & 1.38 \\
2 days after Palmaz stent explantation & 2.90 & 1.22 & 2.18 \\
2 days after Strecker plant implantation & 3.01 & 2.03 & 7.10 \\
13 months after Strecker stent & 2.94 & 1.69 & 5.96 \\
implantation & & & \\
\hline
\end{tabular}

VC: vital capacity; FEV1: forced exiratory volume in one second; PEF: peak expiratory flow. 
a)

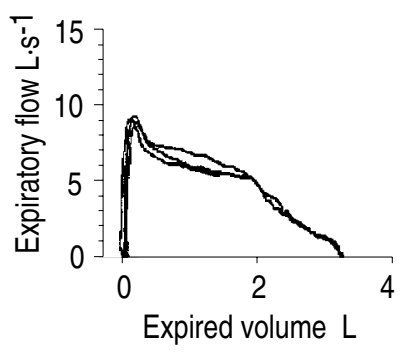

b)

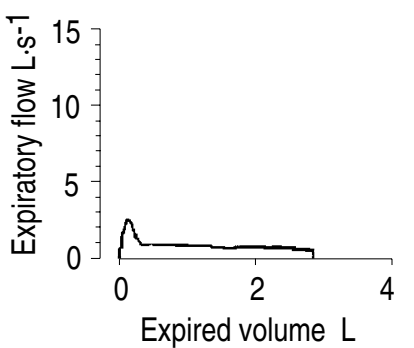

c)

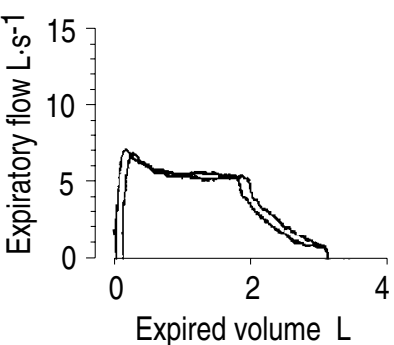

d)

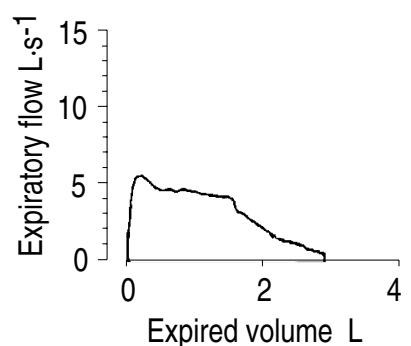

Fig. 1. - Flow-volume diagram: a) 9 months postoperative; b) 45 months postoperative, before Palmaz-Stent implantation when bronchial collapse was present; c) 2 days after Strecker stent implantation; and d) 13 months after Strecker stent implantation.

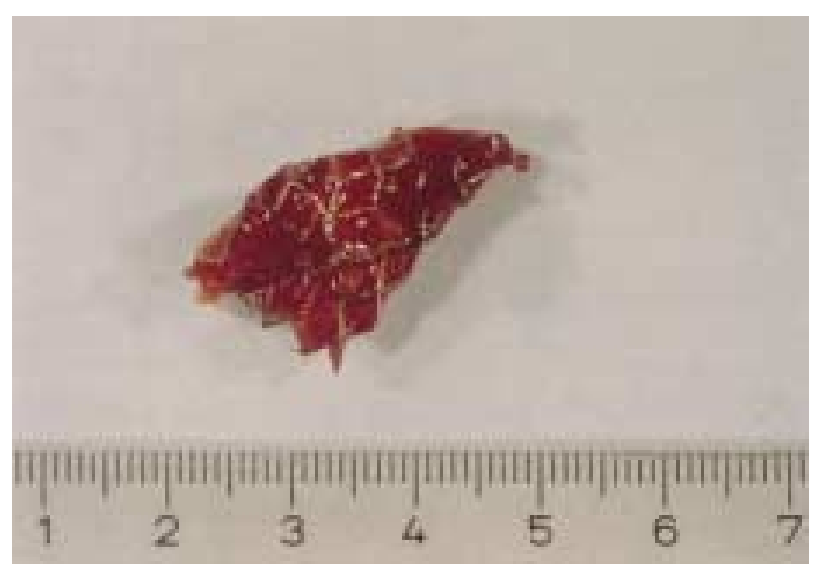

Fig. 2. - Deformed Palmaz-Stent after explantation, blocked by accumulation of purulent secretions.

tional Systems) of axial length $29 \mathrm{~mm}$ and diameter 8 $\mathrm{mm}$ was inserted at another hospital. Subsequently, the patient experienced substantial clinical improvement for $24 \mathrm{~h}$. Thereafter, mucus retention, dyspnoea and cough recurred and worsened markedly within the following 12 days. Two weeks after stent placement, he eventually presented with acute severe dyspnoea, excruciating and insatiable cough, and expectoration of purulent and viscous sputum. FEV1 and PEF had once more dropped to a minimum of $1.02 \mathrm{~L}$ and $1.38 \mathrm{~L} \cdot \mathrm{s}^{-1}$, respectively. Bronchoscopy revealed obstruction of the tracheobronchial anastomosis by a distorted Palmaz stent with accumulation of purulent secretions, which now functioned as a barrier to mucus transport and airflow (fig. 2). An incorrect placement of the stent had been excluded by means of bronchoscopy and radiography. It was noticeable, that deformation was apparent mainly at the distal part of the prothesis. After explantation, an $8 \mathrm{~mm}$ wide and 40 $\mathrm{mm}$ long Strecker-Bronchial-Stent (Boston Scientific Co., Watertown, Massachusetts) was chosen which provided radial compliance and re-expandability. After insertion within the tracheobronchial anastomosis, mucus clearance improved markedly and there was no sign of respiratory distress. FEV1 improved to $2.03 \mathrm{~L}$. The flow-volume diagram regained a near normal shape, with a PEF of $7.10 \mathrm{~L} \cdot \mathrm{s}^{-1}$ (fig. 1c).

At the 13 month follow-up, a decrease in expiratory flow parameters was noted (FEV1 1.69 L; PEF 5.96 L·s $\mathrm{s}^{-1}$ ). Radiographic fluoroscopy revealed a $20 \%$ reduction of the relaxed shape of the stent; however, without noticeable limitation of clinical state. In the lateral view, a reversible shortening of the stent diameter was observed at the very distal end of the prosthesis when the patient was performing voluntary cough manoeuvres. Repeated bronchoscopic evaluation showed satisfactory local tolerance of the Strecker stent without formation of granulomas.

\section{Discussion}

Stenting of unstable airways has been successfully performed using expandable metal stents $[2,9]$. The implantation in unstable airways secondary to bronchial anastomosis has been described in cases of lung transplantation [10,11]. Airflow improvement after stent implantation in benign endobronchial lesions is documented for up to 52 months [8]. Bronchial instability is characterized by expiratory airway collapse, exacerbated by increased respiratory effort, such as exercise or coughing. In this case, bronchial collapse developed secondary to sleeve pneumonectomy and was located at the site of the tracheobronchial anastomosis.

This resulted in the patient's failure to clear accumulated secretions as the cough mechanism plays an important role in tracheobronchial mucus clearance [12]. In patients with hypersecretion of mucus and impaired mucociliary transport in particular, mucus elimination is only compensated for through repeated cough $[13,14]$. When bronchial collapse was present, peak expiratory flow decreased to a quarter of the postoperative value (fig. 1b).

We believe that failure of the Palmaz-Stent in establishing patency of the unstable airway was primarily due to the prosthesis design. Since intrathoracic pressure is transmitted to the surface of the stent without modification by the flaccid airway, elastic recoil, allowing radial dilatation appears to be an indispensable condition in preventing deformation, whereas pronounced stiffness of a prosthesis may result in profound mucosal damage [15]. The importance of mechanical stent characteristics allowing for dynamic compression during cough has recently been shown in an ex-vivo model [16]. The Palmaz-Stent, which consists of a single rigid, slotted, stainless steel tube, offers longitudinal flexibility, whereby radial noncompliance of the component segments is maintained [5]. It is, therefore, assumed that transmitted changes in intrathoracic pressure during cough led to compression and permanent radial deformation, resulting in airway occlusion by accretion of purulent mucus within the def- 
ormed steel tube. An incorrect placement of the Palmaz stent could be excluded by radiological and bronchoscopic evaluation which showed the correct position within the tracheobronchial anastomosis. Accidental damaging of the stent during the implantation procedure appears unlikely since the proximal orifice of the stent, accessible to forceps and bronchoscope, did not show relevant deformation (fig. 2). In addition, substantial symptomatic improvement had been experienced for the first 2 days after placement.

In contrast, the Strecker-Stent is made of alloy filaments braided in the form of a tubular mesh. It appeared to be less rigid, and the inherent expansile properties of the wire allowed for radial dilatation when compressed. After implantation, forced expiratory flow increased considerably. A diffuse airway collapse not limited to the stented segment, would not have shown an improvement in expiratory flow rates after stent placement. The initial drop followed by a plateau as revealed by the flowvolume loop is typical of a dynamic intrathoracic stenosis of the trachea (fig. 1c). This implicates a functional compression of the stent with rising intrathoracic pressure (e.g. forced expiratory manoeuvre, cough). Although maximum radial compression tolerated by the prothesis is limited [6], elasticity to outside compression was sufficient to prevent symptomatic deformation within the period of observation. However, the reduction of the relaxed shape of the stent by $20 \%$ together with a decline in forced expiratory flow parameters 13 months after implantation may be an indication of some deterioration of the structural integrity of the Strecker stent, which may still limit its long-term use. Granulomas or accumulated secretions have not been observed within the stent and do not explain the change in forced expiratory flow. The fluoroscopic observation of diameter shortening only at the distal end of the prosthesis and only in the lateral view is not considered a primary failure of inherent mechanical features of the stent. Although not proven by objective data, it is rather assumed that due to inconsistent structural and functional characteristics of the unstable bronchial segment (operation, radiotherapy) transmission of intrathoracic pressure into force was inhomogenous, and presumably was most effective at the distal part as well as at the anterior and posterior portions, of the prosthesis.

We conclude that, in this case, the implantation of the Strecker stent, a nonrigid prosthesis with limited flexibility, regaining effective cough clearance by avoiding airway collapse represented the major factor in achieving marked symptomatic improvement. Although stenting of unstable airways has been successfully performed, the influence of dynamic compression on the elastic characteristics of metal stents in bronchial collapse has not yet been evaluated in vivo.

In cases of bronchial collapse, elastic recoil, allowing radial dilatation, and firm circular contact with the surrounding bronchial wall to prevent dislocation may be decisive in achieving positive long-term results. This may obviate or reduce the need for early removal. Wire stents seem to meet the specific requirements and can be fashioned according to the expansile properties, length and diameter. It has to be determined whether a self-expandable prosthesis design is more appropriate to maintain expansile force and elasticity in the long-term.

\section{References}

1. Goddard JM, Booker PD. The use of bronchial stents in the management of bronchomalacia. Anaesthesia 1987; 42: $534-535$.

2. Simonds AK, Irving JD, Clarke SW, Dick R. Use of expandable metal stents in the treatment of bronchial obstruction. Thorax 1989; 44: 680-681.

3. Wallace MJ, Charnsangavej C, Ogawa K, et al. Tracheobronchial tree: expandable metallic stents used in experimental and clinical applications. Work in progress. Radiology 1986; 158: 309-312.

4. Mair EA, Parsons DS, Lally KP. Treatment of severe bronchomalacia with expanding endobronchial stents. Arch Otolaryngol Head Neck Surg 1990; 116: 10871090.

5. Fischman D, Savage M, Zalewski A, Goldberg S. Overview of the Palmaz-Schatz stent. J Inv Cardiol 1991; 3: 75-84.

6. Strecker EP, Liermann D, Barth KH, et al. Expandable tubular stents for treatment of arterial occlusive diseases: experimental and clinical results. Work in progress. Radiology 1990; 175: 97-102.

7. Gelb AF, Zamel N, Colchen A, et al. Physiologic studies of tracheobronchial stents in airway obstruction. $\mathrm{Am}$ Rev Respir Dis 1992; 146: 1088-1090.

8. Vergnon JM, Costes F, Bayon MC, Emonot A. Efficacy of tracheal and bronchial stent placement on respiratory functional tests. Chest 1995; 107: 741-746.

9. Nashef SA, Dromer C, Velly JF, Labrousse L, Couraud L. Expanding wire stents in benign tracheobronchial disease: indications and complications. Ann Thorac Surg 1992; 54: 937-940.

10. Egan TM, Westerman JH, Lambert CJJ, et al. Isolated lung transplantation for end-stage lung disease: a viable therapy. Ann Thorac Surg 1992; 53: 590-595.

11. Noirclerc MJ, Metras D, Vaillant A, et al. Bilateral bronchial anastomosis in double-lung and heart-lung transplantations. Eur J Cardiothorac Surg 1990; 4: 314317.

12. Zahm JM, King M, Duvivier C, Pierrot D, Girod S, Puchelle E. Role of simulated repetitive coughing in mucus clearance. Eur Respir J 1991; 4: 311-315.

13. Camner P, Mossberg B, Phillipson K, Strandberg K. Elimination of test particles from human tracheobronchial tree by voluntary coughing. Scand J Respir Dis 1979; 60: 56-62.

14. Puchelle E, Zahm JM, Girard F, et al. Mucociliary transport in vivo and in vitro: relations to sputum properties in chronic bronchitis. Eur J Respir Dis 1980; 61: 254264.

15. Mair EA, Parsons DS, Lally KP. Endotracheal stents for treatment of induced piglet tracheomalacia. Am Coll Surg Forum 1989; 40: 569-571.

16. Freitag L, Eicker R, Linz B, Greschuchna D. Theoretical and experimental basis for the development of a dynamic airway stent. Eur Respir J 1994; 7: 2038-2045. 